\title{
A Stabilized Three-Field Formulation and its Decoupling for Advection-Diffusion Problems
}

\author{
Gerd Rapin ${ }^{1}$ and Gert Lube ${ }^{1}$ \\ Georg-August-Universität Göttingen, Institut für Numerische und Angewandte \\ Mathematik (http://www.num.math.uni-goettingen.de/)
}

\begin{abstract}
Summary. We propose a new stabilized three-field formulation applied to the advection-diffusion equation. Using finite elements with SUPG stabilization in the interior of the subdomains our approach enables us to use almost arbitrary discrete function spaces. They need not to satisfy the inf-sup conditions of the standard three-field formulation. The scheme is stable and satisfies an optimal a priori estimate. Furthermore, we show how the scheme can be solved efficiently in parallel by an adapted Schur complement equation and an alternating Schwarz algorithm. Finally some numerical experiments confirm our theoretical results.
\end{abstract}

\section{Introduction}

In an bounded Lipschitz domain $\Omega \subset \mathbb{R}^{d}, d=2,3$, we consider the problem

$$
L u:=-\epsilon \Delta u+\mathbf{b} \cdot \nabla u+c u=f \quad \text { in } \Omega, \quad u=0 \quad \text { on } \partial \Omega
$$

with $\epsilon>0, \mathbf{b} \in\left(W^{1, \infty}(\Omega)\right)^{d}, c \in L^{\infty}(\Omega)$, and $f \in L^{2}(\Omega)$. Especially the singularly perturbed case $\epsilon<<1$ is of interest, since there the solution can possess sharp layers. Moreover, it is well known, that simple numerical methods fail, since spurious oscillations of the numerical solution may occur.

The three-field formulation was introduced by Baiocchi et al. [1992] (see also Brezzi and Marini [2001]). Decomposing the domain into non-overlapping subdomains the method allows different discretization techniques in different subdomains. Especially, the treatment of non-matching grids is possible. In the discrete case the corresponding function spaces must satisfy two inf-sup conditions. This is quite restrictive for the choice of the discrete spaces. In our stabilized scheme we circumvent these conditions by appending additional terms. The latter terms are well-adapted to the hyperbolic limit $\epsilon=0$.

\section{The three-field formulation}

First let us tackle the global problem (1). The weak formulation reads: 


$$
\text { Find } w \in H_{0}^{1}(\Omega) \mid a_{\Omega}(w, v)=l_{\Omega}(v), \quad \forall v \in H_{0}^{1}(\Omega)
$$

with

$$
a_{G}(w, v)=\epsilon \int_{G} \nabla w \cdot \nabla v d x+\int_{G}(\mathbf{b} \cdot \nabla w+c w) v d x, \quad l_{G}(v)=\int_{G} f v d x
$$

for all $w, v \in H^{1}(\Omega)$ and a domain $G \subset \Omega$. To ensure the well-posedness of (2) we assume the existence of a constant $c_{0}>0$ such that $\tilde{c}:=c-\frac{1}{2} \nabla \cdot \mathbf{b} \geq c_{0}$. Then the Lemma of Lax-Milgram shows that (2) possesses a unique solution.

In order to introduce the three-field formulation, we divide the domain $\Omega$ into $N$ non-overlapping subdomains $\Omega_{i}$ with sufficiently smooth boundaries, i.e. $\bar{\Omega}=\bigcup_{i=1}^{N} \bar{\Omega}_{i}, \quad \Omega_{i} \cap \Omega_{j}=\emptyset, i \neq j$. Moreover, we define local interfaces $\Gamma_{i}:=\partial \Omega_{i} \backslash \partial \Omega$ and the skeleton $\Gamma:=\left(\bigcup_{i=1}^{N} \partial \Omega_{i}\right) \backslash \partial \Omega$. For the three-field formulation three different function spaces are introduced. The first function space $\mathbf{V}:=\prod_{i=1}^{N} V^{i}$ with $V^{i}:=\left\{v^{i} \in H^{1}\left(\Omega_{i}\right)|v|_{\partial \Omega \cap \partial \Omega_{i}}=0\right\}$ is defined on the subdomains. Furthermore, we need a space of Lagrange multipliers $\Lambda^{i}$ on each local interface $\Gamma_{i}$. The local Lagrange multiplier space $\Lambda^{i}$ is given by the dual space of $H_{00}^{\frac{1}{2}}\left(\Gamma_{i}\right)$. We denote the dual product on $\left\langle\Lambda^{i}, H_{00}^{\frac{1}{2}}\left(\Gamma_{i}\right)\right\rangle$ by $\langle\cdot, \cdot\rangle_{i}$. The global space is given by $\Lambda:=\prod_{i=1}^{N} \Lambda^{i}$. The third function space is defined on $\Gamma$ by $\Phi:=\left\{\varphi \in L^{2}(\Gamma)\right.$ : there exists $u \in H_{0}^{1}(\Omega), u=\varphi$ on $\left.\Gamma\right\}$.

Now we formulate the following three-field formulation (cf. Bertoluzza and Kunoth [2000]): Find $\mathbf{u} \in \mathbf{V}, \boldsymbol{\lambda} \in \boldsymbol{\Lambda}$ and $\psi \in \Phi$, such that

$$
\begin{array}{llrl}
\text { i) } \sum_{i=1}^{N} a_{\Omega_{i}}\left(u^{i}, v^{i}\right)-\sum_{i=1}^{N} \epsilon\left\langle\lambda^{i}, v^{i}\right\rangle_{i} & =\sum_{i=1}^{N} l_{\Omega_{i}}\left(v^{i}\right) & & \forall \mathbf{v} \in \mathbf{V} \\
i i) \sum_{i=1}^{N} \epsilon\left\langle\nu^{i}, \psi-u^{i}\right\rangle_{i} & & & \forall \boldsymbol{\nu} \in \boldsymbol{\Lambda} \\
\text { iii }) \sum_{i=1}^{N} \epsilon\left\langle\lambda^{i}, \phi\right\rangle_{i} & & & \forall \phi \in \Phi .
\end{array}
$$

It can be shown that the three-field formulation (3) possesses a unique solution $(\mathbf{u}, \boldsymbol{\lambda}, \varphi) \in \mathbf{V} \times \boldsymbol{\Lambda} \times \Phi$. If the solution $w \in H_{0}^{1}(\Omega)$ of (2) is sufficiently regular, i.e. $\triangle w \in L^{2}\left(\Omega_{i}\right), i=1, \ldots N$, the solution can be represented by

$$
\mathbf{u}=\left(\left.w\right|_{\Omega_{1}}, \ldots,\left.w\right|_{\Omega_{N}}\right), \quad \boldsymbol{\lambda}=\left(\left.\frac{\partial w}{\partial \mathbf{n}_{1}}\right|_{\Gamma_{1}}, \ldots,\left.\frac{\partial w}{\partial \mathbf{n}_{N}}\right|_{\Gamma_{N}}\right), \quad \varphi=\left.w\right|_{\Gamma}
$$

where $\mathbf{n}_{i}$ is the outward normal of $\Omega_{i}$ (cf. Baiocchi et al. [1992]).

\section{A stabilized three-field formulation}

Now the three-field formulation (3) is discretized by linear finite elements. To this end we introduce quasi-uniform meshes $\mathcal{T}_{u}^{i}, \mathcal{T}_{\lambda}^{i}$ and $\mathcal{T}_{\varphi}$ on $\Omega_{i}, \Gamma_{i}$ and $\Gamma$. The meshes can be non-matching. But for simplicity we assume that all 
meshes have the same global mesh size $h$. Moreover, we need the notation $\partial \mathcal{T}_{u}^{i}$ for the restriction of $\mathcal{T}_{u}^{i}$ onto the local interface $\Gamma_{i}$. Thus we obtain discrete spaces $\mathbf{V}_{h} \subset \mathbf{V}, \quad \boldsymbol{\Lambda}_{h} \subset \boldsymbol{\Lambda}$ and $\Phi_{h} \subset \Phi$.

Replacing the continuous function spaces by discrete subspaces, the wellposedness of the discrete scheme of (3) requires two certain inf-sup conditions. One idea to guarantee the conditions is proposed in Brezzi and Marini [2001]. They enrich the space $\boldsymbol{V}_{h}$ by bubble functions. Here, we avoid the constraints by adding some stabilization terms modifying ideas of Baiocchi et al. [1992].

In the advection dominated case further problems occur. Using a standard discretization it is well known that there may arise spurious oscillations of the computed solution. Therefore we use the SUPG method and define

$$
\begin{aligned}
a_{\Omega_{i}}^{S D}\left(u_{h}^{i}, v_{h}^{i}\right) & :=a_{\Omega_{i}}\left(u_{h}^{i}, v_{h}^{i}\right)+\sum_{T \in \mathcal{T}_{u}^{i}} \delta_{T}\left(L u_{h}^{i}, \mathbf{b} \cdot \nabla v_{h}^{i}\right)_{T}, \\
l_{\Omega_{i}}^{S D}\left(v_{h}^{i}\right) & :=l_{\Omega_{i}}\left(v_{h}^{i}\right)+\sum_{T \in \mathcal{T}_{u}^{i}} \delta_{T}\left(f, \mathbf{b} \cdot \nabla v_{h}^{i}\right)_{T}
\end{aligned}
$$

for $i=1, \ldots, N$. The parameter $\delta_{T}$ is defined by $\delta_{T}:=\delta_{0} h_{T}\|\mathbf{b}\|_{L^{\infty}(T)}^{-1}$ in the advection dominated regime for $P e_{T}:=\frac{1}{2} h_{T} \epsilon^{-1}\|\mathbf{b}\|_{L^{\infty}(T)}>1$ and by $\delta_{T}:=\frac{1}{2} \delta_{0} h_{T}^{2} \epsilon^{-1}$ else. Now the error in the interior of the subdomains can be controlled by the streamline diffusion norm

$$
\left\|\left|v_{h}\right|\right\|_{S D, \Omega_{i}}^{2}:=\epsilon\left|v_{h}\right|_{1, \Omega_{i}}^{2}+\left\|\sqrt{\tilde{c}} v_{h}\right\|_{0, T}^{2}+\sum_{T \in \mathcal{T}_{u}^{i}} \delta_{T}\left\|\mathbf{b} \cdot \nabla v_{h}\right\|_{0, T}^{2}
$$

which gives us additional control in the streamline direction. Taking all the mentioned problems into account we end up with the following stabilized three-field formulation: Find $\mathbf{u}_{h} \in \mathbf{V}_{h}, \boldsymbol{\lambda}_{h} \in \boldsymbol{\Lambda}_{h}$ and $\varphi_{h} \in \Phi_{h}$, such that

$$
\begin{aligned}
i) \sum_{i=1}^{N}\left\{a_{\Omega_{i}}^{S D}\left(u_{h}^{i}, v_{h}^{i}\right)-l_{\Omega_{i}}^{S D}\left(v_{h}^{i}\right)-\epsilon\left\langle\lambda_{h}^{i}, v_{h}^{i}\right\rangle_{i}+f_{i}^{-}\left(u_{h}^{i}-\varphi_{h}, v_{h}^{i}\right)\right\} & =0 \\
\text { ii) } \sum_{i=1}^{N}\left\{\epsilon\left\langle\nu_{h}^{i}, u_{h}^{i}-\varphi_{h}\right\rangle_{i}-\sum_{E \in \partial \mathcal{T}_{u}^{i}} \beta_{E}\left(\frac{\partial u_{h}^{i}}{\partial \mathbf{n}_{i}}-\lambda_{h}^{i}, \nu_{h}^{i}\right)_{E}\right\} & =0 \\
\text { iii } \sum_{i=1}^{N}\left\{\epsilon\left\langle\lambda_{h}^{i}, \phi_{h}\right\rangle_{i}-f_{i}^{+}\left(u_{h}^{i}-\varphi_{h}, \phi_{h}\right)\right\} & =0
\end{aligned}
$$

for all $\mathbf{v}_{h} \in \mathbf{V}_{h}, \boldsymbol{\nu}_{h} \in \boldsymbol{\Lambda}_{h}, \phi_{h} \in \Phi_{h}$. We have used the notation

$$
f_{i}^{ \pm}\left(u_{h}^{i}-\varphi_{h}, \psi\right):=\sum_{E \in \partial \mathcal{T}_{u}^{i}} \int_{E}\left(\alpha_{E}+\left(\mathbf{b} \cdot \mathbf{n}_{i}\right)^{ \pm}\right)\left(u_{h}^{i}-\varphi_{h}\right) \psi d s, \quad \psi \in H_{*}^{\frac{1}{2}}\left(\Gamma_{i}\right)
$$

with $\left(\mathbf{b} \cdot \mathbf{n}_{i}\right)^{ \pm}:=\frac{1}{2}\left|\mathbf{b} \cdot \mathbf{n}_{i}\right| \pm \frac{1}{2}\left(\mathbf{b} \cdot \mathbf{n}_{i}\right)$. Thus $\left(\mathbf{b} \cdot \mathbf{n}_{i}\right)^{-}$acts only on the inflow part $\Gamma_{i}^{-}:=\left\{x \in \Gamma_{i} \mid \mathbf{b}(x) \cdot \mathbf{n}_{i}(x)<0\right\}$ and $\left(\mathbf{b} \cdot \mathbf{n}_{i}\right)^{+}$only on the outflow part. The parameters $\alpha_{E}, \beta_{E} \geq 0$ will be specified later.

Let us shortly explain, why we have added the different stabilization terms. $f_{i}^{ \pm}(\cdot, \cdot)$, which are added to the first resp. third line of $(5)$, couple the local spaces $V_{h}^{i}$ and the space $\Phi_{h}$. They give additional control in stream-wise direction, especially in the hyperbolic limit $\epsilon \rightarrow 0$. By $\sum_{E \in \partial \mathcal{T}_{u}^{i}} \beta_{E}\left(\frac{\partial u_{h}^{i}}{\partial \mathbf{n}_{i}}-\lambda_{h}^{i}, \nu_{h}^{i}\right)_{E}$ 
the spaces $\Phi_{h}$ and $\boldsymbol{\Lambda}_{h}$ are coupled. Theses couplings enable us to ignore the mentioned inf-sup conditions.

We can prove the following a priori estimate (for the rather technical proof cf. Rapin and Lube [2003b], Theorems 1 and 2). Here, $a \preceq b$ means, that there exists a constant $C>0$ independent of $h$ and $\epsilon$ such that $a \leq C b$.

Theorem 1. Assume for the stabilization parameters the inequalities

$$
\min \left\{\epsilon / h, \epsilon^{2} / h^{2}\right\} \preceq \alpha_{E} \preceq \max \{1, \epsilon / h\}, \quad \min \left\{\epsilon^{2}, h \epsilon\right\} \preceq \beta_{E} \preceq h \max \left\{h^{2}, \epsilon\right\} .
$$

Then there exists a unique solution $\mathbf{u}_{h} \in \mathbf{V}_{h}, \boldsymbol{\lambda}_{h} \in \boldsymbol{\Lambda}_{h}$ and $\varphi_{h} \in \Phi_{h}$ of (5) and the error is bounded by

$$
\left\|\left|(\mathbf{u}, \boldsymbol{\lambda}, \varphi)-\left(\mathbf{u}_{\mathbf{h}}, \boldsymbol{\lambda}_{h}, \varphi_{h}\right)\right|\right\| \preceq\left(\epsilon^{\frac{1}{2}}+h^{\frac{1}{2}}\right) h \sum_{i=1}^{N}\left|u^{i}\right|_{2, \Omega_{i}}
$$

for a solution $\mathbf{u} \in \mathbf{V} \cap H^{2}(\Omega)$. The norm is given by

$$
\begin{aligned}
\left\|\left|\left(\mathbf{u}_{h}, \boldsymbol{\lambda}_{h}, \varphi_{h}\right)\right|\right\|^{2} & :=\sum_{i=1}^{N}\left(1-\beta_{0}\right)\left\|\left|u_{h}^{i}\right|\right\|_{S D, \Omega_{i}}^{2} \\
& +\sum_{i=1}^{N} \sum_{E \in \partial \mathcal{T}_{u}^{i}} \int_{E}\left[\left(2 \alpha_{E}+\left|\mathbf{b} \cdot \mathbf{n}_{i}\right|\right)\left(u_{h}^{i}-\varphi_{h}\right)^{2}+\beta_{E}\left(\lambda_{h}^{i}\right)^{2}\right] d s
\end{aligned}
$$

If we insert a sufficiently regular solution $(\mathbf{u}, \boldsymbol{\lambda}, \varphi)$ of $(3)$ into the stabilized formulation (5), all additional terms vanish. In this sense (5) is consistent.

There is some degree of freedom for the choice of the stabilization parameters in the advection dominated regime. In the diffusion dominated case we obtain the well known choice of the discontinuous Galerkin method $\alpha_{E} \sim \epsilon / h_{E}$ (and $\beta_{E} \sim \epsilon h_{E}$ ). Using suitable global constants $0<\alpha_{0}, \beta_{0}<1$ we determine

$$
\alpha_{E}=\alpha_{0}\left\{\begin{array}{ll}
\epsilon / h_{E}, & \epsilon \geq h_{E}^{2} \\
\epsilon^{2} / h_{E}^{3}, & \epsilon<h_{E}^{2}
\end{array}, \quad \beta_{E}=\beta_{0}\left\{\begin{array}{ll}
\epsilon h_{E}, & \epsilon \geq h_{E}^{2} \\
h_{E}^{3}, & \epsilon<h_{E}^{2}
\end{array} .\right.\right.
$$

By (7) we mainly enforce boundary conditions in a weak sense on the inflow part of the subdomains, even for $\epsilon=0$.

Remark 1. For given $\varphi_{h} \in \Phi_{h}$ and right hand side $f \in L^{2}(\Omega)$ the equations $(5, \mathrm{i}),(5, \mathrm{ii})$ are discretizations of the local Dirichlet problems

$$
L w^{i}=f \quad \text { in } \Omega_{i} \quad w^{i}=\varphi_{h} \text { on } \partial \Omega_{i}, \quad w^{i}=0 \text { on } \partial \Omega \backslash \partial \Omega_{i} .
$$

These problems are well-posed (cf. Rapin and Lube [2003a]).

\section{A Schur complement method}

Now we derive the corresponding Schur complement equation for our stabilized scheme. Then the solution of (3) can be obtained by solving local problems. Computing the local problems can be done completely in parallel. 
Recall that for given $f \in L^{2}(\Omega), \varphi_{h} \in \Phi_{h}$ the first two lines of (5) are local Dirichlet problems (cf. Remark 1). Denoting the local solutions by $\left(\mathbf{z}_{h}\left(f, \varphi_{h}\right), \gamma_{h}\left(f, \varphi_{h}\right)\right) \in \boldsymbol{V}_{h} \times \boldsymbol{\Lambda}_{h}$ we see

$$
\left(\mathbf{z}_{h}\left(f, \varphi_{h}\right), \gamma_{h}\left(f, \varphi_{h}\right)\right)=\left(\mathbf{z}_{h}(f, 0), \gamma_{h}(f, 0)\right)+\left(\mathbf{z}_{h}\left(0, \varphi_{h}\right), \gamma_{h}\left(0, \varphi_{h}\right)\right)
$$

due to the linearity of the scheme. Inserting this in the third line of (5) yields the Schur complement equation for our scheme: Find $\varphi_{h} \in \Phi_{h}$, such that

$$
\left\langle S_{h} \varphi_{h}, \psi_{h}\right\rangle=\sum_{i=1}^{N}\left\{-\epsilon\left\langle\gamma_{h}^{i}(f, 0), \psi_{h}\right\rangle_{i}+f_{i}^{+}\left(z_{h}^{i}(f, 0), \psi_{h}\right)\right\}, \quad \forall \psi_{h} \in \Phi_{h}
$$

where the discrete Steklov-Poincaré operator $S_{h}$ is defined by

$$
\left.\left\langle S_{h} \varphi_{h}, \psi_{h}\right\rangle:=\sum_{i=1}^{N}\left\{\epsilon\left\langle\gamma_{h}^{i}\left(0, \varphi_{h}\right), \psi_{h}\right\rangle_{i}-f_{i}^{+}\left(z_{h}^{i}\left(0, \varphi_{h}\right)-\varphi_{h}\right), \psi_{h}\right)\right\} .
$$

Theorem 2. The discrete Schur complement equation (8) possesses a unique solution. Moreover, $\left(\mathbf{z}_{h}\left(f, \varphi_{h}\right), \gamma_{h}\left(f, \varphi_{h}\right), \varphi_{h}\right)$ is the solution of (5).

Proof. cf. Rapin and Lube [2003b], Lemma 3.

\section{An alternating Schwarz method}

Tallec and Sassi [1995] describe a non-conforming discretization for the Poisson problem. We extend the algorithm to the advection-diffusion problem using the additional stabilization terms $f_{i}^{ \pm}(\cdot, \cdot)$ of $(5)$. Starting with an initial guess $\left(\psi_{h}\right)_{0} \in \Phi_{h},\left(\lambda_{h}\right)_{0} \in \boldsymbol{\Lambda}_{h}$, we obtain the algorithm:

1. Find $\left(\mathbf{u}_{h}\right)_{k+1} \in \mathbf{V}_{h}$ such that

$$
\begin{aligned}
& a_{\Omega_{i}}^{S D}\left(\left(u_{h}^{i}\right)_{k+1}, v_{h}^{i}\right)+f_{i}^{-}\left(\left(u_{h}^{i}\right)_{k+1}-\left(\psi_{h}\right)_{k}, v_{h}^{i}\right)= \\
& l_{\Omega_{i}}^{S D}\left(v_{h}^{i}\right)+\epsilon\left\langle\left(\lambda_{h}^{i}\right)_{k}, v_{h}^{i}\right\rangle_{\Gamma_{i}}, \quad \forall v_{h}^{i} \in V_{h}^{i} .
\end{aligned}
$$

2. Compute $\left(\lambda_{h}^{i}\right)_{k+\frac{1}{2}} \in \Lambda_{h}^{i}$ by

$$
\epsilon\left\langle\left(\lambda_{h}^{i}\right)_{k+\frac{1}{2}}, \mu_{h}^{i}\right\rangle_{\Gamma_{i}}=\epsilon\left\langle\left(\lambda_{h}^{i}\right)_{k}, \mu_{h}^{i}\right\rangle_{\Gamma_{i}}-f_{i}^{-}\left(\left(u_{h}^{i}\right)_{k+1}-\left(\psi_{h}\right)_{k}, \mu_{h}^{i}\right), \forall \mu_{h}^{i} \in \Lambda_{h}^{i} .
$$

3. Find $\left(\psi_{h}\right)_{k+1} \in \Phi_{h}$ such that there holds for all $\phi_{h} \in \Phi_{h}$

$$
\sum_{i=1}^{N}\left\{\epsilon\left\langle\left(\lambda_{h}^{i}\right)_{k+\frac{1}{2}}, \phi_{h}\right\rangle_{\Gamma_{i}}-f_{i}^{+}\left(\left(u_{h}^{i}\right)_{k+1}-\left(\psi_{h}\right)_{k+1}, \phi_{h}\right)\right\}=0 .
$$

4. Compute $\left(\lambda_{h}^{i}\right)_{k+1} \in \Lambda_{h}^{i}$ such that there holds for all $\mu_{h}^{i} \in \Lambda_{h}^{i}$

$$
\left.\epsilon\left\langle\left(\lambda_{h}^{i}\right)_{k+1}, \mu_{h}^{i}\right\rangle_{\Gamma_{i}}=\epsilon\left\langle\left(\lambda_{h}^{i}\right)_{k+\frac{1}{2}}, \mu_{h}^{i}\right\rangle_{\Gamma_{i}}-f_{i}^{+}\left(\left(u_{h}^{i}\right)_{k+1}-\left(\psi_{h}\right)_{k+1}\right), \mu_{h}^{i}\right) .
$$

It can be proved that the algorithm is well-posed. In step 1 local problems with Robin conditions on the interface are solved. The algorithm is quite similar to the algorithm proposed by Lube et al. [2003]. The Robin values on the inflow part of the local problems are mainly determined by the Robin values of the neighbouring subdomains, computed in the previous step.

A convergence proof of this algorithm is still an open problem. But the numerical results are very promising (cf. Rapin [2003]). 


\section{Numerical experiments}

The main focus of our algorithm is the application to the advection dominated case. Especially the case of nontrivial flows is of interest. To demonstrate the power of our approach we consider the following (quite hard) example.

Example 1. We search for a solution $L u=f$ in $\Omega=(0,1)^{2}$ with boundary conditions $u=-0.5$ on $\gamma_{1}:=\left\{\left(x_{1}, x_{2}\right) \in \partial \Omega \mid x_{2}=0\right\}, u=0.5$ on $\gamma_{2}:=$ $\left\{\left(x_{1}, x_{2}\right) \in \partial \Omega \mid x_{2}=1\right\}$, and $u=0$ on the remainder $\partial \Omega \backslash\left(\gamma_{1} \cup \gamma_{2}\right)$ of the boundary. The flow is given by

$$
\mathbf{b}\left(x_{1}, x_{2}\right):=\left(\left(2 x_{2}-1\right)\left(1-\left(2 x_{1}-1\right)^{2}\right), 4 x_{2}\left(2 x_{1}-1\right)\left(x_{2}-1\right)\right)^{T} .
$$

$\mathbf{b}$ is a rotational flow with a center in $\left(\frac{1}{2}, \frac{1}{2}\right)$ and $\nabla \cdot \mathbf{b}=0$.

We decompose the unit square $\Omega$ into $(6 \times 6)$ squares. In the context of domain decomposition this example is particularly interesting. In the advection dominated case the solution is almost constant in the interior of $\Omega$. The constant is given by the mean value of the Dirichlet data on the boundary. Now any discretization has to find this value by mixing the boundary information.

For a global mesh size $h_{\text {int }}$ the local meshes are chosen by a checkerboard pattern with local mesh sizes $h_{u}=h_{\text {int }} \backslash 2 h_{\text {int }}, h_{\lambda}=(1 / 3) h_{\text {int }} \backslash(2 / 3) h_{\text {int }}$, $h_{\varphi}=h_{\text {int }}$. For all computations we have chosen $\alpha_{0}=1$ and $\beta_{0}=1$. The result for $\epsilon=10^{-6}$ is plotted in Figure 1 (a). It coincides quite well with the solution of the one-domain case. Although the SUPG stabilization technique is used, typically crosswind wiggles of the finite element solution appear.

The purpose of the next example is to numerically validate the a priori estimate of Theorem 1.

Example 2. For $-\epsilon \triangle u+(-1,-1)^{T} \cdot \nabla u=f$ in $\Omega$ and $u=g$ on $\partial \Omega$ we distinguish two cases. (a) We choose $f, g$ in such a way that $u(x, y)=x \cos (\pi y)$ becomes the exact solution. In the second case (b) with $f=1$ and $g=0$ strong boundary layers appear in the singularly perturbed case.

We consider Example 2 (a). Using a decomposition of $\Omega$ into $(6 \times 6)$ subrectangles we alter the mesh size for $\epsilon=1,0.1,10^{-4}$. The results are plotted in Figure 1 (b) and agree with Theorem 1. If we choose the nonsmooth Example 2 (b) with layers, we obtain a convergence rate of $1 / 2$ in the $L^{2}(\Omega)$ norm as in the SUPG case without domain decomposition, since the layers are not resolved. Moreover, we obtain the optimal rates on subdomains $\Omega^{\prime} \subset \Omega$ away from the layers (cf. Rapin [2003]).

Next, we study the effect of the stabilization on the discrete Schur complement equation (8) and the alternating Schwarz algorithm.

We start with the Schur complement equation (8) applied to Example 2 (a). The equation is solved by the GMRES method. In Table 1 (a) we observe that the number of iteration steps is independent of the mesh size for the singularly perturbed case $\left(\epsilon=10^{-4}, 10^{-6}\right)$. In the diffusion dominated regime 

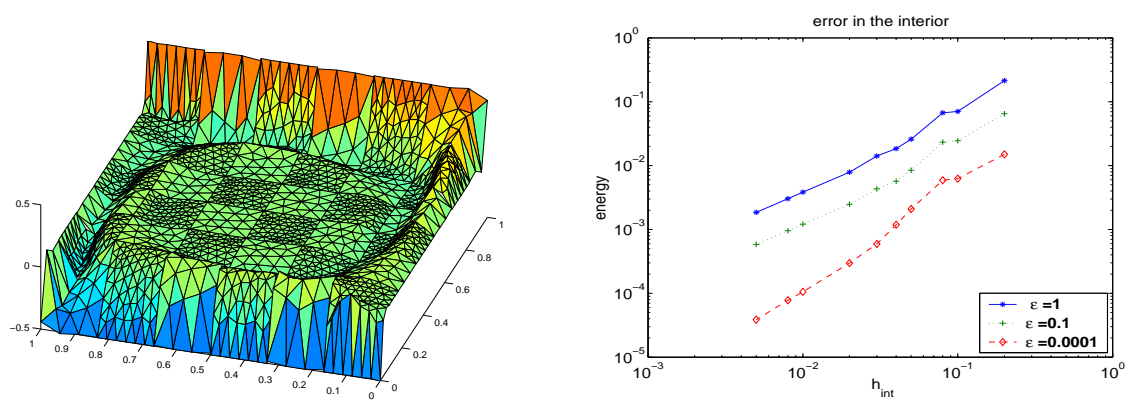

Fig. 1. (a) Plot of Example 1; (b) error in the energy norm $\sum_{i=1}^{N}\left(\epsilon|\cdot|_{1, \Omega_{i}}^{2}+\|\cdot\|_{0, \Omega_{i}}^{2}\right)^{\frac{1}{2}}$ for Example 2 (a).

$(\epsilon=1,0.1)$ the number of iteration steps increases for smaller mesh sizes. Therefore in this case we have to introduce a preconditioner. First experiments with a generalized Neumann-Neumann preconditioner can be found in Rapin [2003]. As expected, in Table 1 (b) we observe an increase of the number of iteration steps for more subdomains.

Please note, that, in general, the local solutions of the first iteration steps possess sharp boundary layers on the outflow part, although the reference solution is smooth. The layers become smaller within the convergence process. Therefore, we obtain the same results for Example 2 (b).

Now we consider the alternating Schwarz algorithm. In our numerical experiments we compare the discrete solution with the reference solution of the continuous problem. In Figure 2 we see that the discretization error is reached within a few steps for the singularly perturbed case. In the diffusion dominated case the convergence is quite slow, but can be accelerated by an adaptive choice of the parameter $\alpha_{E}$ (cf. Lube et al. [2003]).

Summarized one can state that both methods work well both in the diffusion dominated case and the singularly perturbed case. But we suggest to

\begin{tabular}{||l||l|l|l|l||}
\hline$\epsilon \backslash h_{\text {int }}$ & 0.05 & 0.02 & 0.01 & 0.005 \\
\hline \hline 1 & 21 & 32 & 46 & 66 \\
\hline $10^{-1}$ & 19 & 31 & 43 & 59 \\
\hline $10^{-4}$ & 19 & 20 & 19 & 18 \\
\hline $10^{-6}$ & 19 & 20 & 19 & 19 \\
\hline \multicolumn{4}{|c}{ (a) }
\end{tabular}

\begin{tabular}{||l||l|l|l|l|l|l||}
\hline$\epsilon \backslash n$ & 2 & 4 & 6 & 8 & 10 & 12 \\
\hline \hline 1 & 25 & 51 & 65 & 78 & 81 & 97 \\
\hline $10^{-1}$ & 26 & 21 & 26 & 30 & 34 & 38 \\
\hline $10^{-4}$ & 16 & 21 & 26 & 30 & 34 & 38 \\
\hline $10^{-6}$ & 6 & 21 & 26 & 30 & 34 & 38 \\
\hline \multicolumn{5}{|c|}{ (b) }
\end{tabular}

Table 1. Number of iteration steps of the GMRES algorithm, which is needed to reduce the initial residuum by the factor $10^{-8}$ for Example 2 (a). The initial guess is always 0 . In (a) we consider different mesh sizes $h_{\text {int }}$ and diffusion coefficients $\epsilon$ for a $(4 \times 3)$ partition. In (b) the domain is decomposed into $(n \times n)$ subdomains for mesh size $h_{\text {int }}=0.01$. 

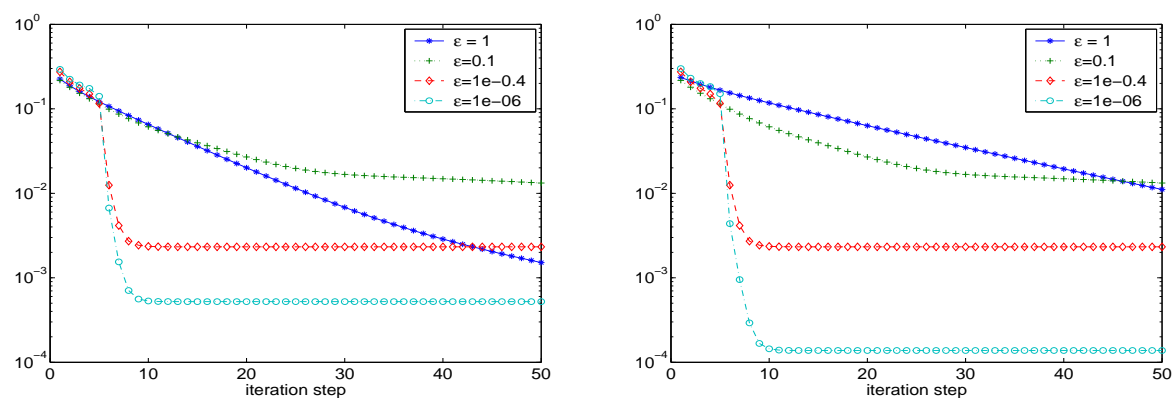

Fig. 2. Convergence behavior of the alternating Schwarz algorithm in the $L^{2}(\Omega)$ norm for a $(4 \times 3)$ decomposition. On the left hand side the mesh size is chosen by $h_{\text {int }}=0.02$ and on the right hand side by $h_{\text {int }}=0.01$.

use the Schur complement method in the diffusion dominated case and the alternating Schwarz method in the advection dominated case.

\section{References}

C. Baiocchi, F. Brezzi, and L. Marini. Stabilization of Galerkin methods and applications to domain decomposition. In A. Bensoussan and J. Verjus, editors, Future Tendencies in Computer Science, Control and Applied Mathematics, pages 345-355, Berlin-Heidelberg-New York, 1992. SpringerVerlag.

S. Bertoluzza and A. Kunoth. Wavelet Stabilization and Preconditioning for Domain Decomposition. IMA J. Numer. Anal., 20:533-559, 2000.

F. Brezzi and D. Marini. Error Estimates for the three-field formulation with bubble stabilization. Math. Comp., 70:911-934, 2001.

G. Lube, T. Knopp, and G. Rapin. Acceleration of a non-overlapping Schwarz method for advection diffusion problems. Technical report, Universität Göttingen, 2003. DD 15 Preprint.

G. Rapin. The Three-field Formulation for Elliptic Equations: Stabilization and Decoupling Strategies. PhD thesis, Universität Göttingen, 2003.

G. Rapin and G. Lube. A stabilized scheme for the Lagrange multiplier method for advection-diffusion equations. Technical report, Universität Göttingen, 2003a. appears in $M^{3} A S$.

G. Rapin and G. Lube. A stabilized scheme of the three-field approach for advection-diffusion equations. Technical report, Universität Göttingen, 2003b. submitted.

P. L. Tallec and T. Sassi. Domain Decomposition with nonmatching grids: Augmented Lagrangian Approach. Math. Comp., 64:1367-1396, 1995. 